\title{
Patrice Chéreau, metteur en scène de Shakespeare
}

\author{
Catherine Treilhou-Balaudé
}

\section{(2) OpenEdition \\ Journals}

\section{Édition électronique}

URL : http://journals.openedition.org/shakespeare/638

DOI : 10.4000/shakespeare.638

ISSN : 2271-6424

Éditeur

Société Française Shakespeare

Édition imprimée

Date de publication : 1 novembre 2000

Pagination : 193-208

ISBN : 2-84269-407-4

\section{Référence électronique}

Catherine Treilhou-Balaudé, «Patrice Chéreau, metteur en scène de Shakespeare », Actes des congrès de la Société française Shakespeare [En ligne], 18 | 2000, mis en ligne le 01 novembre 2007, consulté le 02 mai 2019. URL : http://journals.openedition.org/shakespeare/638; DOI : 10.4000/shakespeare.638 


\section{S H A K E S P E A R E \\ \& L A F R A N C E}

Société Française Shakespeare

Actes du Congrès de 2000

* * *

Textes réunis et présentés par

Patricia DORVAL

publiés sous la direction de Jean-Marie MAGUIN 
Colloque honoré d'une subvention du Ministère de l'Éducation Nationale, de la Recherche et de la Technologie

Site Internet $:<$ http://alor.univ-montp3.fr/SFS/> Liste de diffusion : <sfs-1@smr1.univ-montp3.fr>

Tous droits de traduction, de reproduction et d'adaptation réservés pour tous les pays.

(C) 2000. Société Française Shakespeare, École Normale Supérieure, 45 rue d'Ulm, 75005 Paris.

ISBN 2-84269-407-4 


\section{P A T R I CE CHÉREAU, METTEUR EN S C E N E DE S H A K E S P E A R E}

Le Prix de la révolte au marché noir, pièce de Constantin Dimitriadis, montre les étudiants d'une troupe universitaire essayant de monter tant bien que mal des scènes de Shakespeare. Ayant mis en scène cette pièce, durant la saison 1968-1969, au théâtre de la Commune d'Aubervilliers, Patrice Chéreau, venu lui-même au théâtre, quelques années auparavant, par la troupe étudiante du lycée Louis-le-Grand, disait à son propos qu'elle décrivait la mort d'une certaine forme de théâtre et la nécessité d'inventer un nouvel usage du théâtre. Moins de deux ans plus tard, sa mise en scène de Richard II invite à penser qu'il a trouvé, ou qu'il est en train de trouver, ce nouvel usage qui lui permettra, à plusieurs reprises, de porter sur la scène Shakespeare, Marlowe, ou même Molière, sans pour autant risquer la fossilisation du théâtre.

De fait, Chéreau n'a plus cessé de se confronter au théâtre de Shakespeare, jusqu'après son retrait de l'institution théâtrale, puisque c'est sur des extraits de 3 Henry VI et Richard III qu'il a fait travailler les élèves de troisième année du Conservatoire dans l'atelier qu'il a dirigé en 1998, dont les travaux ont abouti à un spectacle public. Si ses rencontres avec Shakespeare n'ont pas été très fréquentes, elles se sont révélées marquantes : l'Hamlet de 1988 est un spectacle décisif dans l'histoire des mises en scène de Shakespeare en France. Après les avoir rappelées, nous observerons les fils directeurs de l'approche scénique de Shakespeare par 
Chéreau, en partant de l'espace, toujours premier, pour aller vers les dimensions du théâtre de Shakespeare qu'il approfondit dans chacun de ses spectacles : le théâtre de l'histoire, le théâtre comme artifice et fabrique d'illusion, et enfin le théâtre de la conscience.

Patrice Chéreau a vingt-cinq ans lorsqu'il monte Richard II, dans la traduction française de Pierre Leyris ${ }^{1}$, au Nouveau Gymnase à Marseille en 1970, avant son départ pour le Piccolo Teatro de Milan. Le spectacle sera repris à l'Odéon-Théâtre de France en 1971. Suite à la défection d'un acteur, Chéreau interprète lui-même le rôle de Richard II, pour lequel il est dirigé par un autre comédien du spectacle, Daniel Emilfork. Gérard Desarthe, futur Hamlet, interprète pour l'heure le rôle de Bolingbroke, Pierre Asso celui de Jean de Gand. Le décor est créé en collaboration avec Richard Peduzzi, présenté à Chéreau par Jacques Schmidt en 1968. Ce dernier crée les costumes, et André Diot est aux éclairages. L'équipe artistique qui œuvrera ensemble pendant deux décennies est déjà constituée.

En 1972, alors que Roger Planchon a proposé à Chéreau la codirection artistique du théâtre de la Cité, à Villeurbanne, Chéreau met en scène Le Massacre à Paris, de Marlowe, dans une adaptation de Jean Vauthier.

En 1975, il monte Lear, d'Edward Bond, toujours à Villeurbanne, et le reprend à Paris, à l'Odéon-Théâtre de France. Des détracteurs lui reprochent d'avoir préféré au vieux roi shakespearien ce tyran moderne, qui fait entourer son royaume d'une muraille dont la construction met à nu la violence de l'oppression de la classe ouvrière. Pourtant, ce choix est caractéristique de l'approche du théâtre élisabéthain qui est celle de Chéreau à cette époque, anti-archéologique, passant par la recherche d'enjeux contemporains.

En 1984 a lieu une présentation semi-publique des travaux de la première promotion des élèves de l'École du Théâtre des Amandiers, que Chéreau a créée avec Pierre Romans. Ce dernier a travaillé sur Tchekhov et Goldoni, et Chéreau sur quatre comédies de Shakespeare: Beaucoup de bruit pour rien, Peines d'amour perdues, La Nuit des Rois, Comme il vous plaira.

Il s'agit de l'unique, bref, mais intéressant contact de Chéreau avec l'univers des comédies shakespeariennes, qu'il déclare avoir ignoré jusqu'à ce moment. Il les choisit pour leurs personnages qui 
ont gardé des liens avec leur adolescence, évitant aux jeunes gens qu'il fait travailler toute forme de composition. Ces comédies nécessitent de rendre crédibles les rapports entre des êtres au comportement contradictoire, des personnages qui se transforment au contact les uns des autres.

En juillet 1988, le théâtre des Amandiers de Nanterre, que dirigent Patrice Chéreau et Catherine Tasca, est très présent au $42 \mathrm{e}$ festival d'Avignon.

Chéreau monte Hamlet au festival d'Avignon, dans la traduction d'Yves Bonnefoy, avec Gérard Desarthe dans le rôle-titre, Luc Bondy présente Le Conte d'hiver, dans une traduction de Bernard-Marie Koltès. Daniel Emilfork propose La Journée des chaussures, de Cosmo Clarke, et Pierre Romans un spectacle à partir du montage de divers textes de Tchekhov. Enfin, Dans la solitude des champs de coton, de Koltès est repris dans une usine désaffectée, avec Patrice Chéreau dans le rôle du dealer.

Ce programme est révélateur de la manière dont Shakespeare s'inscrit dans le répertoire de Chéreau: Shakespeare est présent à des moments-clés, et la Cour d'honneur en est un, mais il n'est pas seul, pas plus qu'il n'est isolé dans un passé lointain d'où il s'agirait de l'exhumer : il côtoie Koltès, il le rencontre même, avec la traduction du Conte d'hiver. Des liens se tissent entre toutes ces créations. Dans un entretien, Chéreau dit être longtemps passé à côté d'Hamlet, et y être venu de, ou par, Platonov, qu'il a monté l'année précédente en Avignon. Il semble que Chéreau se tourne vers Shakespeare à des moments où son théâtre lui offre matière à approfondir des questions ou des thèmes qu'il trouve au même moment dans des textes plus contemporains : le mécanisme de la prise du pouvoir au début des années 70 (note sur la pièce de Neruda), la solitude et l'opacité des relations inter-personnelles au moment d'Hamlet et de la création des textes de B.-M. Koltès.

Hamlet et Dans la solitude des champs de coton sont d'ailleurs les deux mises en scène que Chéreau reprendra à plusieurs reprises ${ }^{2}$ et dans divers lieux.

Il peut s'agir aussi de parentés d'ordre théâtral. Hamlet doit à Platonov, peut-être plus encore que la thématique générale du mal d'être et de l'incommunicabilité, l'approfondissement d'un travail sur le rythme : variations nombreuses de la vitesse, enchainements rapides des séquences, effets de rupture entre les différents moments de la fable. 
Depuis que Patrice Chéreau s'est retiré de l'institution théâtrale en 1990, et tourné vers le cinéma, il est revenu quelquefois au théâtre : pour reprendre Dans la solitude des champs de coton, et le plus récemment, en se tournant à nouveau vers Shakespeare et vers l'enseignement et la transmission, fonction décisive du théâtre. Il disait déjà en 1984, à propos de son travail avec les élèves de Nanterre :

Il $\mathrm{y}$ a un moment de la vie où il faut aller à la rencontre de ceux qui viennent après nous dans le métier. C'est un élan obligatoire, quelque chose de beau, d'inévitable et qui exige un peu d'humilité. ${ }^{3}$

Et il constatait que vingt ans d'âge le séparaient de ces jeunes comédiens. Pour 3 Henry VI, Richard III, fragments, atelier d'élèves de $3 \mathrm{e}$ année du Conservatoire présenté à la Manufacture des EEillets d'Ivry du 10 au 22 novembre 1998, l'écart s'est encore creusé, mais l'élan s'est renouvelé et approfondi : cette fois, Chéreau n'hésite pas à travailler sur la composition, en distribuant à de jeunes comédiennes de vingt-cinq ans les rôles de la reine Marguerite et de la duchesse d'York, et même celui d'Édouard, prince de Galles. Il n'hésite pas non plus, dans ce contexte plus libre que celui d'une production normale, à «tailler» dans les pièces choisies la fable qui l'intéresse: il part des scènes sanglantes de la guerre civile anglaise, retenant les épisodes extrêmes, tels les lamentations parallèles d'un père et d'un fils sur le corps, le premier de son fils et l'autre de son père, qu'ils ont tués de leurs propres mains sur le champ de bataille ${ }^{4}$, pour clore le spectacle au sommet de l'ascension de Richard Gloucester, à l'acte IV de Richard III.

Les choix opérés par Chéreau dans l'œuvre de Shakespeare, mais aussi la manière dont ces choix s'inscrivent à l'intérieur d'un répertoire plus vaste, dans une tension permanente avec des auteurs plus contemporains, font apparaître l'intérêt de Chéreau pour la dimension historique et politique du théâtre shakespearien et élisabéthain : la représentation d'événements traumatiques de l'histoire, comme la déposition et l'assassinat d'un roi ou les massacres générés par une guerre civile, est articulée à un souci constant de leur signification contemporaine. Le choix de l'adaptation modernisante du Massacre à Paris par Vauthier, ou de la réécriture du Roi Lear par Bond, permet l'investissement de figures archaïques (celle du roi Lear), ou d'événements lointains (le 
massacre de la Saint-Barthélémy) par des problématiques actuelles : le transfert du pouvoir du politique sur l'économique, la violence toujours actuelle des conflits idéologiques. Dans le cas de Richard II, c'est la mise en scène qui porte seule l'actualisation du sens, la réflexion sur le mécanisme de la prise du pouvoir, non sans faire violence au classicisme de la traduction de Pierre Leyris, qui manifeste publiquement ses réserves.

Les trois autres rencontres avec Shakespeare se font dans une perspective assez nettement différente: l'exploration fragmentaire et expérimentale de l'univers des comédies est menée dans un cadre plus pédagogique qu'artistique; l'approche d'Hamlet, plus philosophique et poétique que politique, est convergente avec le choix de la traduction d'Yves Bonnefoy. Enfin, Richard III, que Chéreau souhaitait déjà monter à l'époque d'Hamlet, apparaît vraiment comme une synthèse des rencontres précédentes, avec l'importance accordée par Chéreau à. la dimension politique de la fable, ainsi qu'à l'enjeu métaphysique du mal et de sa force de séduction, qui passe par l'exploration des pouvoirs du théâtre, ainsi que par la liberté dramaturgique que donne la forme fragmentaire.

Pour tous ses premiers spectacles, Patrice Chéreau a travaillé d'abord sur des idées d'espace, et conçu lui-même ses décors, avant de s'adjoindre, à peu près au moment de Richard II, la collaboration d'un peintre, Richard Peduzzi. Par la suite, l'espace est resté une donnée première du travail de Chéreau avec les comédiens. À l'exception de Henry VI/Richard III, réalisé dans des conditions scénographiques minimales, les espaces conçus pour le théâtre de Shakespeare présentent des caractéristiques communes. Nous partirons donc de l'espace des mises en scène shakespeariennes de Chéreau, donnée première et support de la présence et du jeu des comédiens, pour nous interroger ensuite sur les aspects de la théâtralité shakespearienne que Patrice Chéreau exalte de manière récurrente dans ses mises en scène.

La mise en scène de Shakespeare s'accompagne de l'abandon de la structure à l'italienne chère à Chéreau. Ce fait n'est pas seulement dû à la contrainte de la frontalité, commune à la Cour d'Honneur, à la grande salle des Amandiers de Nanterre et à la manufacture des Eillets. Le choix, pour Shakespeare, d'un espace unique, soumis seulement à des variations mécaniques à vue, et qui 
signifie aussi bien le dedans que le dehors, relève de l'esthétique scénique de Chéreau ainsi que d'une représentation spécifique non tant de la Renaissance que de la tension entre l'époque historique et culturelle dont relève le théâtre shakespearien, et la nôtre. Dans cet espace aux temporalités multiples sont confrontés de manière dynamique l'élément ou le matériau naturel - terre, eau, bois - et les signes de l'activité humaine, physis et technè.

Un véritable feuilletage des références historiques se donne à lire dans l'espace et dans les costumes. L'espace est celui de l'histoire, d'une histoire qui se joue dans un monde traversé (ou bouleversé) par l'action des hommes.

Le moyen âge et la Renaissance, par quoi Shakespeare et ses fables dramatiques appartiennent à un temps lointain, sont toujours cités, plus ou moins fortement, par des références architecturales, picturales, dans l'espace et souvent dans les costumes. La façade palladienne qui forme le fond de Richard II est projetée sur le sol dans Hamlet. Les problèmes de civilisation, de verticaux (la question de la sacralité du roi) deviennent horizontaux et même souterrains: Hamlet illustre le passage du moyen âge à la Renaissance, que Patrice Chéreau, suivant l'interprétation d'Yves Bonnefoy, comprend comme

celui de la religion à la conscience, où l'on découvre que le destin est solitude et que la nature est matière.

Il ajoute aussitôt :

Il faut en même temps trouver tout ce que cela peut nous dire, à nous, à une époque de doute, où les certitudes s'effritent. ${ }^{5}$

La modernité est toujours présente également, par la référence, généralement stylisée, à des pratiques contemporaines : les poulies et les grues de Richard II renvoient à notre industrialisation en même temps qu'aux machines rêvées à la Renaissance, les immeubles peints du Massacre à Paris, à la construction du Front de Seine, contemporaine de la mise en scène de cette pièce. Pour Henry VI/Richard III, le lieu même est marqué par l'activité industrielle. Dans Hamlet, ce sont les comédiens, en costumes et 
chapeaux mous style années 50, qui semblent sortir de Platonov, ou d'un film de Fellini, tandis que les autres costumes évoquent picturalement la Renaissance.

Les comédiens d'Hamlet portent de petites valises ressemblant à celles dont ne se séparaient jamais Richard II et ses suivants, dixhuit ans plus tôt. Chéreau pratique l'auto-citation, un peu à la manière de Truffaut. Dans les trois spectacles, des intrusions musicales très actuelles (Pink Floyd, Prince), renforcent la modernité de la gestuelle. Certains passages de la bande-son d'Hamlet, et notamment les percussions électriques qui ponctuent les venues du spectre, sont repris dans Henry VI/Richard III, signes discrets de reconnaissance, d'une mise en scène de Shakespeare à l'autre.

Une seconde caractéristique commune de l'espace des mises en scène shakespeariennes consiste dans la confrontation d'un matériau naturel ou élémentaire et des signes, architecturaux ou mécaniques, de l'activité humaine ou de la vie sociale.

Le plateau de Richard II, entièrement recouvert de sable, évoque une fosse que les comédiens ne foulent que rarement, la plupart des scènes se déroulant sur des plate-formes de bois de différentes hauteurs, situées sur les côtés du plateau et manœuvrées par des treuils. La présence de ce sable ne ressemble pas à celle, malgré tout illustrative, de l'argile sableuse qui jonchera le chantier de Combat de nègre et de chiens de Koltès, en 1983. Dans Richard II, ce sable est symbolique : matériau minéral mouvant et volatil, cour basse pour Richard descendu rejoindre Bolingbroke, espace tauromachique pour la mise à mort du roi déchu, il retrouve son antique valeur d'usage, éponger le sang et la sueur dans les arènes, les stades, les cirques. Douze ans après ce spectacle mémorable, dans son article Le blanc $d u$ roi, François Regnault se souvient encore que ce sable, dans lequel Richard II courait pieds nus lors de la scène de la déposition, «éclaboussait les cheveux des premiers rangs» ${ }^{6}$.

Bernard Dort, dans un article important consacré aux débuts de l'activité théâtrale de Chéreau, décrit ainsi l'espace scénique de Richard II :

Richard II va se dérouler dans une sorte de fosse (la cour intérieure d'un palais féodal mais aussi une arène destinée aux combats de 
fauves). ${ }^{7}$

«Ceci mais aussi cela»: cette cohabitation du lieu référentiel d'une fable historique, et d'un lieu second, déjà théâtral, sera récurrente dans les mises en scène de Chéreau. Le sable de Richard II introduit l'élémentaire dans le machiné, mais la présence du naturel n'intéresse pas Chéreau en tant que telle: il ne figure pas, en effet, le jardin du duc d'York ${ }^{8}$, irruption dans la pièce de Shakespeare d'une nature ordonnée et immédiatement lisible. Dans la pièce (note : acte III scène 4), n'intéresse pas Chéreau, qui ne la figure pas.

Dans la mise en scène du Massacre à Paris, l'eau qui recouvre entièrement le plateau, plombant les costumes des comédiens, se révèle un peu plus illustrative, puisqu'elle fait référence à la Seine et à ses quais, théâtre du massacre de la Saint-Barthélémy, mais toujours fortement symbolique. L'eau transforme la scène en cloaque, en espace de délitement et de putréfaction où flottent des cadavres ${ }^{9}$.

Dans Hamlet, le bois marqueté de la façade Renaissance renversée sur le sol, qui se hérisse et s'enfonce tout au long de la représentation, rappelle bien sûr le matériau du théâtre élisabéthain, et de tout plateau. Grâce à la mobilité des éléments, on retrouve aussi l'effet de sol mouvant, qui se dérobe, qui caractérisait Richard II comme Le Massacre à Paris. Le sol qui se dérobe, mettant en danger l'acteur et son mouvement, est une constante très juste des mises en scènes shakespeariennes de Chéreau, pour symboliser une époque où l'on remet en cause, dans l'inquiétude, toutes les certitudes, notamment celle de la terre ferme et plate, tandis que la verticalité de l'espace métaphysique est toujours prégnante.

Il s'agit d'un bois à la fois travaillé par l'architecture, par l'histoire qui s'y inscrit, la Renaissance, et porteur, sur le plateau, d'une nature primitive, par sa densité, sa résistance à l'eau, au vent, aux forces élémentaires auxquelles s'expose tout décor en Avignon. Cet aspect est renforcé par l'apparition saisissante et sonore, sur ce bois, du cheval sombre et farouche monté par le spectre. L'élémentaire porté sur le théâtre signifie immédiatement et comme primitivement, dans un «en-deçà» du langage qu'a décrit admirablement Yves Bonnefoy ${ }^{10}$.

Contiguë à l'élément, la machine fonctionne également comme un symbole, non comme une simple image ou une 
métonymie de la praxis humaine. Elle reste une invention de théâtre, qui énonce l'esprit et non la lettre de notre modernité.

Les trappes, ponts-levis, treuils, cordages de Richard II configurent un espace complètement machiné dans sa dimension verticale, image de l'activité fébrile des hommes en quête de pouvoir et de la maîtrise des rouages d'une société en pleine mutation.

Une verticalité inverse caractérise Hamlet, dont le sous-sol se fait tour à tour monde des spectres, des acteurs de théâtre qui s'y enfoncent lors de la représentation, des machinistes qui le parcourent en tous sens, actionnant, invisibles, des leviers semblables à ceux que Richard II exhibait. «Le décor induisait fatalement la présence de tout un monde qui grouillait en dessous, qui ressortait», se souvient, dix ans plus tard Richard Peduzzi ${ }^{11}$.

Enfin, l'ossature de la manufacture des Eillets, laissée nue, demeure le squelette métallique d'une activité industrielle abandonnée.

Dans cette tension entre le matériau et la machine, l'espace, mobile et dangereux, s'offre essentiellement au mouvement des corps, à une gestuelle de type baroque comme au mouvement de la fuite, car l'espace du théâtre élisabéthain, tel que le conçoit Chéreau, est toujours un espace piégé.

Le théâtre devient une épreuve pour les comédiens : le sable, véritable «théâtrogramme», entrave les déplacements, marque les pas, porte les traces du poids, du mouvement, de l'effort. L'eau suggère la noyade, d'ailleurs des corps y flottent, à la fin du Massacre à Paris.

Le plateau d'Hamlet, avec ses trappes innombrables et sa pente, était l'instrument d'une mise sous tension décisive pour le jeu des comédiens.

Hamlet pointe du doigt le sol sur lequel il se tient lui-même lorsqu'il déclare que le théâtre est le piège où il prendra la conscience du roi : tout est déjà théâtre dans Hamlet, selon Chéreau, il n'y a pas de «degré zéro» de réalité de la pièce, ou de sol plus ferme que celui du théâtre.

Dans Henry VI/Richard III, la contrainte et le danger sont portés par le corps même des comédiens, et suggérés par l'occupation de l'espace : mouvements en tous sens, effrénés, entre les séquences, entrées et sorties en courant...

L'image des acteurs, seuls ou groupés, sillonnant le plateau de leur démarche agitée, souvent en diagonale, est récurrente dans les 
spectacles de Chéreau.

La mise en scène de Richard II proposait une véritable composition, dans l'espace, des rapports de force et des actions. Bernard Dort ${ }^{12}$ y voyait exposée la rivalité de deux groupes humains, les comédiens, soit Richard II et sa suite, maquillés de blanc, mimique et gestuelle théâtralisées à l'extrême, et les lutteurs, Bolingbroke et ses partisans, qui ne connaissent que la force et prennent le dessus sur les premiers.

Avec les jeunes comédiens du Conservatoire, Chéreau a poussé à l'extrême ce travail choral sur les groupes humains et leur investissement de l'espace, mené également dans Hamlet, qui s'apparente à la prise de possession ou à l'invasion d'un territoire : ce sont les corps-boulets lancés dans les batailles de 3 Henry VI, images brutes de la barbarie toujours sur le point d'affleurer derrière les conventions des relations sociales. Il ne s'agit jamais de la figuration mimétique d'une bataille mais de la métaphore nue des relations entre les hommes : une lutte à mort, une chorégraphie de la haine, que l'on retrouve de la Saint-Barthélémy du Massacre à Paris, à celle, cinématographique, de la Reine Margot.

Ce mouvement choral, qui définit l'espace de civilisation dans lequel évoluent les personnages shakespeariens, est rythmé par une bande-son le plus souvent violente, paroxystique : tangos des années vingt, airs déchirants chantés par la Callas pour Richard II, musiques de hard-rock pour Hamlet et Henry VI/Richard III. Ce mouvement paroxystique caractérise aussi la pantomime de l'acte III d'Hamlet, qui donne l'image d'un théâtre onirique, cauchemardesque, mettant à nu, jusqu'à l'outrance, la violence qui régit les rapports humains. Même parodique, il s'agit néanmoins d'un écho de la pratique théâtrale de Chéreau lui-même.

Mis à l'épreuve dans l'espace-piège, le corps de l'acteur se trouve également mis en péril dans le contact de l'autre. Ce qui distingue l'approche de Shakespeare par Chéreau, et peut-être l'approche de tout texte par Chéreau, c'est l'expression, dans le corps de l'acteur, dans le contact des corps entre eux, dans le geste fondamental de "prendre-aimer-tuer» l'autre, d'une relation fantasmatique entre les personnages. Cette relation des corps en vient parfois à balayer en quelque sorte celle, plus policée, que l'échange verbal établit. La rencontre entre Hamlet et le spectre participe à l'évidence de ce double niveau d'adresse du spectacle au spectateur : au moment du récit détaillé de la mort de son père, dans la fleur de ses péchés, Hamlet est à genoux et se bouche les 
oreilles en secouant la tête, et l'image du corps qui ne supporte pas de recevoir le fardeau répugnant des fautes du père se superpose à un discours filial plus mesuré. On pourrait évoquer aussi la séquence $^{13}$ au cours de laquelle Ophélie rapporte à son père la récente visite d'Hamlet, hors de lui et muet. Son récit, précis et appliqué, tient de la confession d'une fille obéissante et qui n'a rien à cacher à son père ; cependant elle prononce ces mots en se tordant sur le sol et en mimant de son corps l'abandon au désir : le corps contredit la représentation de soi en vierge impassible que donne la parole.

Le corps dit ce que les mots de Shakespeare ne disent pas mais approchent ou laissent deviner, la violence du désir et son ambivalence, celle-là propre à Chéreau : désir d'étreinte, désir de mort. Les corps figurent alors un autre niveau de la fable et des événements qui la composent, que la seule interprétation du texte, aussi intelligente soit-elle, ne permet pas, ou n'atteint pas.

À l'exception de la parenthèse pédagogique à l'école de Nanterre, Chéreau s'est toujours tourné vers l'histoire et vers la tragédie: comme d'autres metteurs en scène, il s'intéresse à Shakespeare ou à Marlowe pour leurs enjeux de pensée, en même temps que pour une certaine grandeur de l'histoire faite théâtre, portant sur la scène des questions et des événements à la fois fondateurs et signifiants pour le présent, telle la question de la légitimité du pouvoir et de sa transmission, ou les maux de la guerre civile.

Dans le théâtre de Shakespeare, Chéreau choisit des œuvres qui racontent le passage violent d'une époque à une autre, qui associent crise des valeurs et crise de la représentation. On trouve dans les trois pièces de son répertoire shakespearien un ou des rois assassinés, et deux fratricides. Les images thêâtrales fortes et violentes que proposent les spectacles de Chéreau sont alors la transposition visuelle d'événements fondamentaux plutôt que la restitution mimétique des événements particuliers dont la fable est tissée. L'esthétique scénique baroque de Chéreau, toute de paroxysme dans l'image, dans le cri, d'oppositions violentes du clair et de l'obscur, rivalise avec l'écriture shakespearienne plus qu'elle ne l'illustre ou ne la sert.

En même temps, chaque mise en scène réfléchit sur le pourquoi et le comment des événements de la fable : Richard II montre, selon Chéreau, 
[...] le passage d'un État, qui, dans son anarchie de nature, se met en opposition avec la classe au pouvoir elle-même, à la naissance historique d'un pragmatisme politique, à l'exigence d'un pouvoir fort autocratique. ${ }^{14}$

La mise en scène de Chéreau visualise dans l'espace les rapports de force, et l'élimination, par la classe dirigeante, du souverain dans lequel elle ne se reconnaît plus. L'une des images les plus marquantes est celle de la déposition : Richard est saisi par une grue sur une passerelle située à douze mètres du sol, et ramené, en bas, dans la fosse.

Hamlet commence en quelque sorte où s'achève Richard II : le roi étant déjà tué, la pièce montre ce qui arrive alors, mais sur un plan métaphysique autant et plus que politique, et d'un autre point de vue que de celui du nouveau roi, quoique l'on observe aussi dans le personnage de Claudius, attentivement construit par Chéreau, les mêmes tourments de l'âme que ceux d'Henry IV à la fin de Richard II, qui torturent ceux qui ne peuvent grandir qu'aspergés de sang.

Le personnage de Richard III, auquel Chéreau s'intéressait dès le moment d'Hamlet, accomplit l'itinéraire complet, l'ascension par le meurtre, l'accès grisant à la royauté et la chute, inévitable châtiment de celui traître à Dieu et à son propre sang. Chéreau ouvre donc le spectacle sur le meurtre d'Henry VI par Richard Gloucester. Cependant, il l'interrompt significativement avant la déchéance du roi meurtrier, et le spectacle s'achève sur l'apothéose de Richard III.

Ce spectacle clôt ainsi de manière ambiguë un cycle historique ouvert avec Richard II. Le programme de Henry VI/Richard III présente d'ailleurs le dernier spectacle dans la continuité du premier : «le crime originel dont l'expiation s'achève» avec l'extinction de la querelle dynastique entre York et Lancastre est la déposition du roi Richard II en 1399. Chéreau aura donc exposé l'origine et le dénouement de ce drame, ou plutôt sa dernière et fascinante figure, à vingt ans de distance.

L'histoire est également théâtre, dans la vision de Shakespeare qui est celle de Chéreau, en ce qu'elle met aux prises des hommes qui jouent et d'autres qui ne jouent pas, entre lesquels passe la frontière, évidemment intéressante pour un metteur en scène, de la 
conscience ou de l'ignorance de la théâtralité inhérente à l'histoire. Il y a dans les pièces de Shakespeare que choisit Chéreau une théâtralité fondée sur l'artifice, sur la pratique consciente de l'illusion : Richard II, Hamlet, Richard III, et certains de ceux qui les entourent sont des comédiens expérimentant les pouvoirs du théâtre. Le dernier, par la jubilation du faux dont il empreint toute la pièce, où le vice le plus noir se donne avec succès l'apparence de la vertu, semble le frère des personnages des Paravents ou de Dans la solitude des champs de coton. Chéreau choisit dans Shakespeare une image du thêâtre comme artifice trompeur, où ce qui «semble», comme dit Hamlet, coïncide rarement avec ce qui est, de préférence à d'autres pièces (Le Conte d'hiver, Mesure pour mesure, ou même Comme il vous plaira), où le théâtre permet plutôt de démasquer les apparences trompeuses en évitant la mort des héros.

Le Richard II de Patrice Chéreau n'est pas déchiré entre la fonction royale et une identité intime dépressive et défaillante, mais il interprète le personnage royal (en le tournant d'ailleurs vers le grotesque) comme un rôle de théâtre. Derrière le clown tragique, il n'y a rien d'autre que la conscience de jouer. Une image forte de ce vide essentiel est fournie par l'interprétation scénique de la fameuse séquence du miroir : l'objet demandé par Richard II à l'acte IV est figuré par un cadre de bois à l'intérieur duquel Richard saute à pieds joints, vers le public. Il n'y a pas de distinction entre le soi et l'image de soi, le soi est déjà image, image de roi mise en doute par la facétie.

Richard II se dit et se montre comme un personnage jouant tour à tour le roi et le mendiant, un personnage dont la faillite se lit dans le masque blanc et le corps désarticulé. La frontière passe ainsi entre ceux qui jouent, amers et lucides, la comédie du pouvoir (Richard et sa suite), et ceux qui agissent et ne jouent pas (Bolingbroke et ses partisans). Ceux-là considèrent les enjeux économiques et politiques tels la stabilité du royaume appuyée sur un pouvoir fort, la défense et l'intégrité de la propriété individuelle, comme des valeurs qui n'appellent pas la mise à distance du doute. Néanmoins, à la fin de la pièce, Bolingbroke-Desarthe adopte des mimiques et une gestuelle semblables à celles de Richard IIChéreau : inévitablement, le nouveau roi devient l'acteur de la fonction royale.

Dans Hamlet, la cour d'Elseneur est montrée comme l'espace d'une théâtralité forcenée, souvent farcesque : ce sont les entrées des personnages royaux et des courtisans, à grands pas, envahissant 
le plateau au rythme d'une musique rock assourdissante, se retournant en faisant virevolter leurs manteaux ; c'est l'interprétation expressionniste de Bernard Ballet-Polonius, sur-jouant le rôle du père et celui du courtisan. C'est Hamlet lui-même, face aux mauvais acteurs qui singent la grandeur royale (Claudius) ou l'amitié (Rosencrantz et Guildenstern), qui se contorsionne et grimace, leur renvoyant une image dégradée et grotesque de leur propre jeu. Cet effet de miroir le dispense d'ailleurs de feindre la folie, puisqu'il suffit à Hamlet de jouer de manière consciente l'artifice des relations sociales pour sembler très différent de ceux qui jouent sans savoir qu'ils jouent.

Ce théâtre facétieux, jubilant de son artificialité, est pourtant dangereux. Hamlet dans le même temps met en œurre et subit le pouvoir d'illusion du théâtre : il trompe par le théâtre, mais son théâtre l'abuse lui-même, ou le comble. Le monologue d'Hécube le montre, plus histrion que jamais, mimant la mise à mort de Claudius avec l'épée de bois laissée là par un comédien, prêt à se satisfaire de cette vengeance théâtrale. Ainsi que l'analyse Yves Bonnefoy dans la préface de la traduction d'Hamlet ${ }^{15}$ qu'il a revue pour la mise en scène de Chéreau, il ne croit peut-être plus lui-même aux valeurs anciennes qu'il est chargé, par le spectre, de maintenir.

Richard III éprouve également avec une joie de comédien le pouvoir du jeu : celui de fabriquer une image de soi totalement fausse, mais qui se donne, et qui est reçue, pour vraie. Le théâtre est le lieu du miracle désespérant de cette tromperie. En cours de répétition ${ }^{16}$, Chéreau conseille ainsi Jérôme Huguet, le jeune comédien interprétant Richard, pour ce passage :

«Plongez au fond de mon cœur, pensées, voici Clarence», situé au sein d'une séquence du spectacle qui enchaîne sans rupture la scène 5 de l'acte $V$ de 3 Henry VI, au cours de laquelle Richard Gloucester tue Henry VI à la Tour et projette l'élimination de Clarence, et la première scène de Richard III.

Gloucester va jouer le frère, le bon frère, dit Chéreau, il faut là un changement phénoménal, il faut impérativement que le mal ait le visage du bien, il n'y a à l'œil aucune différence entre lui et un honnête homme, sinon c'est trop facile. 
Dans le spectacle, l'acteur, face au public, passe sa main sur son visage, le recouvrant du masque invisible de la vertu.

Parvenir à faire prendre le mal pour le bien est une faculté troublante du théâtre. Mettre en scène Richard III revient, pour Chéreau, à explorer une figure du mal absolu et sa force de séduction, par-delà bien et mal.

Mais le théâtre histrionique et jubilatoire que l'on joue dans la sphère sociale contamine également le rapport à soi, ou peut-être symbolise le fait qu'entre soi et soi, il y a toujours, irrémédiablement, du jeu. Richard II, Hamlet, et Richard III partagent, dans l'interprétation qu'en donne Chéreau, une forme de théâtralité tragique de l'intérieur. Ces trois pièces sont ponctuées de monologues auxquels les mises en scène de Chéreau accordent une importance capitale : l'acteur, seul, dans un halo lumineux qui le détache des ténèbres, projette face au public une parole libérée du dialogue. Hamlet, surgi on ne sait d'où, arpente l'immense plateau d'Avignon ou de Nanterre, enjambe les marches qui le hérissent, s'assied, se couche, parle et joue le théâtre de son rapport à soi.

Richard Gloucester, immédiatement après l'assassinat d'Henry VI, sort en claudicant de l'ombre, où il a traîné le cadavre du roi :

Puisque l'enfer a ainsi façonné mon corps, que l'enfer, en réponse, me torde l'âme.

C'est l'origine d'un thêâtre de soi, pour soi. Richard Gloucester est celui qui tente de dire lui-même et pour lui-même qui il est :

Je suis sans frères, pareil à aucun frère, ce mot amour, que les vieux sages trouvent divin et bon pour leurs pareils, mais pas pour moi, je suis moi seul, moi-même. ${ }^{17}$

Chéreau montre sa solitude absolument nue, en même temps que l'énergie désespérée à dire, nommer sa singularité absolue, à l'éloigner de lui en la disant, tente de capter, par le visage de l'acteur mais aussi par un travail quasi-musical de diction, le geste de se représenter à soi. On est très loin à la fois d'un quelconque démonisme du personnage qui s'essaierait auprès du public à une captatio benevolentiae, ou d'un fonctionnement mélodramatique par lequel le traître avertirait le public de sa nature maléfique, avant de 
feindre la vertu et l'humanité... Chéreau montre au contraire l'authenticité de Richard Gloucester au cœur même de la théâtralité de sa représentation de soi, le moment où tout homme disant «je suis», en parlant, sent son être se dérober, se soustraire à sa parole, et ce qu'il dit, le nommer et le manquer à la fois. Hamlet et Richard avant de jouer devant les autres, sont les comédiens d'eux mêmes, se projettent dans la parole, et découvrent que c'est déjà un autre que l'on montre en le disant, altérité inévitable, tragique, et essentielle qui définit à la fois l'humanité, et la nécessité du théâtre.

Ce moment trouve évidemment sa dimension, son ampleur métaphysique dans le passage par un corps et par une voix, dans cette redondance belle et désespérée : nous sommes au théâtre, nous jouons et nous reflétons le théâtre qui est en nous, qui est nous.

Ce que Shakespeare offre spécifiquement à un homme de théâtre comme Patrice Chéreau, c'est sans doute cette coexistence, non de l'épique et du dramatique, mais d'une théâtralité manifeste et d'une théâtralité intériorisée, reflet d'une représentation moderne du sujet dissocié qui est encore la nôtre.

Catherine TREILH OU-B AL AUDÉ Université Paris III - Sorbonne Nouvelle

\section{N O T E S}

${ }^{1}$ Publiée dans l'édition complète du théâtre de Shakespeare au Club Français du Livre, en 1957.

${ }^{2}$ Hamlet : création au $42 \mathrm{e}$ festival d'Avignon, Cour d'Honneur, 919/07/1988, puis tournée en France durant l'automne 1988 (TNP de Villeurbanne, Clermont-Ferrand, Grenoble), grande salle du théâtre des Amandiers de Nanterre 29/11/88 - 12/02/89, puis tournée internationale à l'automne 1989 : Moscou, Berlin, Milan, Francfort, Barcelone. Et enfin reprise pour 20 représentations à la Grande Halle de la Villette, 123/12/1989. 136 représentations au total, devant 149000 spectateurs.

${ }^{3}$ Entretien au Monde, 4 octobre 1984.

${ }^{4} 3$ Henry VI, II.5.55-122. 
${ }^{5}$ Entretien au Nouvel Observateur, 7-14/07/1988.

${ }^{6}$ Les Voies de la création théâtrale, volume XIV : «Chéreau», textes réunis et présentés par Odette Aslan, Paris, éditions du C.N.R.S., 1986, p. 82.

${ }^{7}$ Bernard Dort, «Patrice Chéreau ou le piège du théâtre», in Théâtre réel, Paris, Seuil, 1971, p. 104-11.

${ }^{8}$ Richard II, III.4.

${ }^{9}$ On trouvera un souvenir très prégnant de ce décor dans le spectacle Great Britain adapté d'Édouard II de Marlowe, et mis en scène par JeanHugues Anglade aux Amandiers de Nanterre, au printemps 1984.

${ }^{10}$ Yves Bonnefoy, «Le lieu, l'heure, la mise en scène», in Nanterre Amandiers, les années Chéreau, 1982-1990, Paris, Éditions de l'Imprimerie Nationale, 1991.

11 «Dans les dédales de bois d'une façade projetée au sol», entretien avec Richard Peduzzi réalisé par Samra Bonvoisin, in Hamlet, La Nuit des rois. Shakespeare, la scène et ses miroirs, Théâtre aujourd'hui $\mathrm{n}^{\circ} 6$, Paris, C.N.D.P., 1998, p. 107.

${ }^{12}$ Dans l'article cité note 7.

${ }^{13}$ Hamlet, II.1.78-101.

${ }^{14}$ Programme de Richard II, reproduit partiellement dans Les Voies de la création thêatrale, volume XIV : «Chéreau», op. cit., p. 23-4.

${ }^{15}$ Hamlet est publié avec Le Roi Lear, traduits par Yves Bonnefoy, aux éditions Gallimard, collection Folio, en 1988. La traduction d'Hamlet reprend celle de l'édition du Club Français du Livre (1957), et la préface est celle de l'édition Gallimard de 1978.

${ }^{16}$ Voir l'intéressant documentaire en cinq parties de Stéphane Metge diffusé en décembre 1999 sur ARTE, La leçon de théâtre, Patrice Chéreau, Henry VI/Richard III, 3e partie : le monologue.

17 Henry VI, V.5.78-84. Traduction D. Loayza. 